\title{
Repensando la Promoción de la Salud en el Sistema General de Seguridad Social en Salud
}

\author{
Juan C. Eslava-Castañeda \\ Médico. M. Sc. en Sociología. Departamento de Salud Pública, Facultad de \\ Medicina, Universidad Nacional de Colombia. \\ E-mail: solracnauj2@yahoo.com; jceslavac@unal.edu.co
}

Recibido 4 Junio 2006/Enviado para Modificación 14 Junio 2006/Aceptado 15 Octubre 2006

\section{RESUMEN}

El artículo presenta un balance acerca de la manera como se ha venido entendiendo la Promoción de la Salud (PS) en el ámbito internacional y señala que, cuando menos, existen tres distintas acepciones de la promoción: la primera como orientación general de política, la segunda como conjunto de acciones y la tercera como una dimensión especial del trabajo sanitario. En medio de las sutiles diferencias que se establecen desde estas diversas maneras de asumir la PS, surge el interés expreso por darle una fundamentación específica que la distinga de la prevención y que trascienda la labor educativa. Una vez reconocido el reto que plantea la PS en el campo sanitario, el texto describe de forma sucinta, cómo se introduce el discurso de la PS en Colombia y discute la forma como se ha intentado darle concreción dentro del Sistema General de Seguridad Social. Por último, se hace mención de los esfuerzos que actualmente se están haciendo en el país dirigidos a hacer de la Promoción de la Salud una realidad en el sistema sanitario colombiano.

Palabras Clave: Promoción de la salud, política de salud, seguridad social, Colombia (fuente: DeCS, BIREME).

\section{ABSTRACT}

Rethinking how health is promoted in the Colombian general healthrelated social security system (Sistema General de Seguridad Social en Salud)

This article takes stock of how promoting health $(\mathrm{PH})$ (promoción de la salud - PS) has been understood within an international setting and gives three meanings for promotion: as general policy orientation, as a set of actions 
and as a special dimension of sanitary work. Interest expressed in giving it a specific basis distinguishing it from prevention, transcending educational work, has emerged from the subtle differences established from such different ways of coming to terms with $\mathrm{PH}$. After recognising the challenge posed by $\mathrm{PH}$ in the field of health, the text succinctly describes how discourse regarding $\mathrm{PH}$ has been introduced in Colombia and discusses how an attempt has been made to be more precise regarding its place in the general healthrelated social security system. Efforts currently being made in Colombia regarding making $\mathrm{PH}$ become a reality within the Colombian health system are then mentioned.

Key Words: Promoting health, health policy, social security, Colombia (source: $\mathrm{MeSH}, \mathrm{NLM}$ )

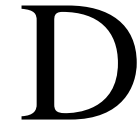

esde mediados de los años 80 del siglo XX la Promoción de la Salud (PS) ha sido ampliamente reconocida como corriente innovadora en el campo de la salud y referente internacional. Tanto así que, pese a las diferentes orientaciones teóricas que se han propuesto y las diversas modalidades que ha adquirido su concreción práctica, hoy en día nadie discute acerca de la importancia que la PS tiene para la labor sanitaria, si bien se admite la necesidad de adelantar procesos sistemáticos que permitan una evaluación seria de sus resultados (1-3).

Este reconocimiento se evidencia en la sucesión de Conferencias Internacionales que, desde 1986, han brindado las orientaciones generales en el campo de la PS y han servido como referentes fundamentales para erigir a la promoción como un derrotero a seguir. De hecho, las grandes organizaciones sanitarias internacionales, así como una gran cantidad de países, han creado estructuras institucionales para el desarrollo de la PS y han reorganizado su propia labor incorporando los lineamientos emanados de dichas Conferencias Internacionales así como los avances en la investigación y en la puesta en práctica de experiencias específicas en promoción (4-5).

Sin embargo, el gran despliegue que ha tenido y el arduo trabajo teórico y operativo que se ha desarrollado a su alrededor no ha eximido a la PS de cierta ambigüedad en el abordaje y de la presencia de algunos equívocos frente a su misma definición y puesta en marcha.

En especial, resalta la confusión que, por momentos, se ha tenido entre la promoción de la salud y la acción preventiva, lo cual ha llevado a menospreciar la importancia de la PS o a refrenar sus potencialidades. Igualmente, 
cabe señalar la tendencia a equiparar PS con educación sanitaria lo cual, a su vez, ha llevado a limitar los alcances de la acción promocional.

Con todo, cada vez es más claro que la PS adquiere una consistencia propia ligada a las nociones de bienestar y calidad de vida y es asumida como un proceso social y político que posibilita la construcción colectiva de la capacidad para proteger y mantener la salud de las poblaciones (6).

Ahora bien, al intentar sintetizar las diversas definiciones que se han elaborado en los últimos años, se puede caracterizar la PS desde tres acepciones diferentes, pero complementarias:

1. Como una orientación de política sanitaria a nivel internacional que busca intervenir sobre el entorno y modificar los estilos de vida posibilitando con ello que las opciones más saludables sean las más fáciles de elegir.

2. Como un conjunto de acciones y procesos encaminados a que las comunidades y los individuos estén en condiciones de ejercer un mayor control sobre los determinantes de la salud manteniendo o mejorando, de ese modo, su «estado de salud».

3. Como una dimensión del trabajo en salud, que toma como eje la salud en sentido positivo -y no la enfermedad- $\mathrm{y}$ tiene por objeto mantener las condiciones de salud y garantizar condiciones de bienestar de los individuos y las poblaciones.

Tomando como base estas acepciones, se hace entendible el por qué la PS se ha planteado, a veces indiscriminadamente, como dimensión, como estrategia, como proceso o como conjunto de acciones (7-9).

En la actualidad, se puede decir que la visión que más peso tiene es aquella que entiende a la PS como un referente general para las políticas de salud y estrategia fundamental de la acción sanitaria. Como estrategia, la PS está encaminada a proporcionar a los pueblos los medios necesarios y suficientes para mejorar su salud y ejercer un efectivo control sobre la misma y, como lo señala Nutbeam, "La promoción de salud constituye una estrategia que vincula a la gente con sus entornos y que, con vistas a crear un futuro saludable, combina la elección personal con la responsabilidad social” (9).

Entre los principios centrales de la PS cabe señalar, por su importancia, los siguientes: la labor de la PS implica a la población en su conjunto y en el contexto de su vida cotidiana; se centra en la acción sobre los determinantes de la salud; combina métodos diversos y complementarios para incidir tanto 
a nivel de los individuos como de las políticas públicas y aspira a la participación efectiva de la población (10).

Esto implica que toda acción en PS exige un planteamiento frente a los determinantes de la salud. De manera habitual, los profesionales del área de la salud adoptan el esquema del campo de salud donde se mencionan cuatro grandes categorías de determinantes: el componente biológico, el medio ambiente, los estilos de vida y los sistemas sanitarios (asistenciales). Sin embargo, se ha reconocido que este esquema tiene algunos problemas y, en la práctica, ha llevado a dos grandes distorsiones: por un lado, ha permitido que el componente de los estilos de vida se magnifique y desfigure hasta el punto de favorecer una visión descontextualizada de los factores de riesgo individuales que llega a «culpabilizar a la víctima» e imposibilita una comprensión de la significación potencial de los procesos que operan sobre la salud a nivel colectivo. Por otro lado, y a pesar de su expreso intento por trascender esa visión, ha seguido fortaleciendo la mirada de la salud desde el referente de los servicios asistenciales a la enfermedad

Es por ello que se han planteado grandes discusiones frente a la PS y se han ido formulando diversos esquemas para su abordaje, con el propósito explícito de superar estas distorsiones y lograr que la PS supere definitivamente el concepto biomédico de salud y dirija su accionar a la construcción de opciones saludables para la población en general.

En lo que hace referencia a la manera como se ha intentado darle concreción a la PS, vale la pena recordar que la Carta de Ottawa (1986) estableció las bases que han servido de marco de referencia para la acción y definió cinco áreas de actuación que, hasta el momento, han guiado la labor de la PS. Estas cinco áreas son: la formulación de políticas públicas saludables, la creación de ambientes que favorezcan la salud, el desarrollo de las habilidades personales, el refuerzo a la acción comunitaria y la reorientación de los servicios de salud.

A partir de este planteamiento inaugural, la labor emprendida ha intentado transformar en propuestas más concretas estas orientaciones. En lo que atañe a la formulación de políticas públicas saludables, este tema fue el eje central de la Segunda Conferencia Internacional sobre PS (Adelaida, 1988) y desde entonces se ha convertido en uno de las más importantes tópicos de trabajo en PS. Aquí se han usado como ejemplos lo acontecido en Noruega con relación a su política de nutrición y alimentación y en Finlandia con el Proyecto de Carelia del Norte. Igualmente se ha relevado la experiencia Canadiense como una propuesta importante a estudiar $(5,11)$. 
Las otras áreas también han sido desarrolladas pero, hay que reconocerlo, de manera bastante desigual. El tema de los entornos saludables fue abordado como eje central de la Tercera Conferencia Mundial sobre PS (Sundsval, 1991) y las propuestas que más amplio reconocimiento han tenido tal vez sean las de Ciudades saludables y Escuelas saludables mientras que, con la paulatina participación de los países pobres, el tema de la equidad en salud se ha convertido en tema fundamental $(6,10,12)$.

Pero si bien la perspectiva de la PS como estrategia internacional ha primado y, en gran medida, ha subsumido las otras acepciones, internamente dos aspectos siguen siendo motivo de tensión: por un lado, la articulación de la tercera manera de entender la PS arriba señalada frente a las otras dos y, por otro lado, la articulación del trabajo que busca transformaciones sociales con el que se enfoca en el cambio de los comportamientos individuales.

La manera en que se han intentado resolver estas tensiones, además de otras, ha sido privilegiando (y reforzando) un discurso general acerca del vinculo entre PS, equidad y desarrollo, asumiendo que el objetivo de la PS es crear las condiciones que garanticen el bienestar general de los pueblos y permitiendo el despliegue de múltiples trabajos de alcance diverso. De esta manera, si bien cierta ambigüedad permanece, tácitamente se asume que la PS recoge la labor preventiva y que la labor del cambio comportamental y de los estilos de vida debe ser complementaria (y armonizada) con la labor que busca cambios significativos en la sociedad.

A la dinámica misma de desarrollo de los diversos trabajos se le ha venido agregando, como criterio orientador, por lo menos para el caso de América Latina, el reconocimiento de la imposibilidad de restringir, en un marco único, la riqueza de la PS en la Región. De esta manera, se admite la necesidad de desarrollar marcos múltiples para el desarrollo conceptual y metodológico de la PS (4).

\section{La Promoción de la Salud en Colombia}

En Colombia, el discurso de la PS empezó a cobrar vitalidad a comienzos del decenio de los noventa, cuando se recogieron los principios formulados en la Carta de Ottawa y se enunciaron como parte de la política de salud, bajo la programática de los “municipios saludables”. Este vínculo entre PS y municipios saludables es señalado de manera explícita por el Ministerio de Salud de la época pero también queda de manifiesto en la Declaración para la Promoción de la Salud que fue adoptada por los participantes del Foro so- 
bre Descentralización y Municipios Saludables, llevado a cabo en la ciudad de Santafé de Bogotá en diciembre de 1991, donde se identifican a las "políticas saludables", los "estilos de vida”, el "ambiente”, la "participación comunitaria", las "desigualdades en salud" y la "convivencia, solidaridad y democracia” como los campos de acción de la PS (13-14).

De manera más clara y con una legitimad aún mayor, el discurso de la PS fue impulsado con la realización de la Conferencia Internacional de Promoción de la Salud en Bogotá, a finales del año 1992. Allí se estableció como tema central (y requisito fundamental) el logro de la equidad y se planteó como el gran desafío de la PS en la América Latina la transformación social que posibilite conciliar los intereses económicos con la búsqueda del bienestar y la equidad social (15).

Pese a este claro llamado, la orientación de la PS se vio alterada debido a los efectos de la reforma sectorial de salud que se formuló a finales del año 1993. Dicha reforma transformó el sector a partir de un esquema de aseguramiento que creo instituciones aseguradoras, redefinió el papel de las instituciones prestadoras de servicios y creo paquetes básicos de servicios. En este contexto, se ubicó a la PS como una función importante a ser desarrollada pero según un esquema organizativo que dividió tajantemente las acciones colectivas de las acciones individuales y que originó no pocas dificultades (7).

De esta manera, el nuevo Sistema General de Seguridad Social (SGSSS) estableció condiciones, recursos y espacios para el desarrollo de la PS. En particular, la formulación de un Plan de Atención Básica (PAB) con varias líneas de acción abrió posibilidades para el desarrollo de actividades dirigidas a las colectividades que fuesen articuladas desde el ámbito territorial. Mientras que, por otra parte, la creación de las Empresas Promotoras de Salud mostró un cierto interés por asegurar que acciones de PS se pudieran implementar en la acción frente a los individuos

Desde la normatividad emitida a partir del año 1997, se definieron una serie de servicios, procedimientos y suministros que el SGSSS debe garantizar a las personas aseguradas con el propósito de mantener y recuperar su salud. Estos incluyen acciones de PS que las aseguradoras deben ofrecer a la población afiliada, en los grupos poblacionales de niños, niñas y adolescentes, y la tercera edad, y en los ámbitos de la salud sexual y reproductiva, la convivencia pacífica y la salud mental. 
De ésta manera, y ya dentro del SGSSS, la PS empezó a ligarse de manera muy fuerte con una orientación de trabajo de educación para el cambio de comportamientos y, bajo el criterio de que la salud y la enfermedad son inseparables, se adoptó un enfoque que, si bien formalmente, vio la PS de forma complementaria a la acción preventiva, en el desarrollo práctico fusionó la PS con la prevención. De hecho, en nuestro medio se acuñó la expresión "promoción y prevención" cuya unión, representada sintéticamente por la sigla P\&P, intenta resolver -aunque de hecho lo oculta- el problema teórico, conceptual y operativo que subyace en la diferenciación de estos dos ámbitos de acción.

De esta manera, si bien la PS en el SGSSS intentó recoger la tradición que viene desde la Carta de Ottawa, reforzada por la Declaración de Santafé de Bogotá, al desplegarla en acciones prácticas le dio un énfasis al componente que tiene que ver con el desarrollo de aptitudes personales y los cambios en los comportamientos individuales. Y asociado a ello, le otorgó una orientación preventivista a la acción promocional, de tal manera que las acciones de P\&P quedaron inscritas en una estrategia de información, educación y comunicación que estimula la construcción de hábitos saludables referidos al control de factores de riesgo y favorece la educación para el buen uso de los servicios preventivos, como mecanismo de inducción a la demanda.

Este énfasis explica el por qué la PS tiene una específica orientación en nuestra labor cotidiana como profesionales de salud y el por qué, por momentos, resulta difícil aplicar toda la riqueza del trabajo en PS dentro del esquema forjado por el SGSSS. El reto que se tiene, por tanto, es abrir las posibilidades para que se lleve a cabo un adecuado y efectivo trabajo promocional dentro de un contexto que, por tradición local, ha pensado más en prevención que en promoción.

Un reto abierto para el futuro inmediato

En los últimos años ha surgido en el país la necesidad de revisar la manera como el SGSSS aborda la labor de P\&P y de propiciar una regulación más precisa de la PS, dado que, tal como se ha venido afirmando en algunos escenarios, no se han definido de manera clara estándares básicos de calidad y cobertura que caractericen el tipo de servicios en materia de promoción de la salud a los que tienen derecho los usuarios del SGSSS.

Podría decirse que esta necesidad ha sido, en parte, fruto de los discusiones internas hechas por el Ministerio de la Protección Social referidas a la 
evaluación del sistema de aseguramiento y, en parte, resultado de una realidad obstinada que permanentemente pone en evidencia importantes problemas de calidad, oportunidad y eficiencia en la labor de cada uno de los actores institucionales del complejo SGSSS.

Una de las maneras como se ha intentado responder a esta situación ha sido mediante la propuesta de elaboración de unas guías de PS, referidas a poblaciones y ámbitos específicos, los cuales ya están estipulados normativamente: salud de niños (as) y adolescentes, salud sexual y reproductiva, salud en la tercera edad, convivencia pacífica con énfasis en el ámbito intrafamiliar, desestímulo del consumo de alcohol y sustancia psicoactivas, y condiciones sanitarias del ambiente intradomiciliar.

Las Guías, que en la actualidad están en la última fase de elaboración, buscan enfrentar el reto de hacer realidad la acción promocional dentro del SGSSS, intentando con ello incorporar los logros y experiencias de la PS al esquema de aseguramiento creado en Colombia. Por supuesto, esto exige un delicado proceso de ajuste y articulación que permita abrir el paso a la PS en un entorno difícil pero expectante y con anhelos de innovación.

Aquí será fundamental mantener como referente la idea de la PS como una dimensión del trabajo sanitario cuyo centro está en la noción de salud, en su sentido positivo como bienestar y calidad de vida, que se articula de múltiples formas con la acción preventiva y que combina acciones individuales y colectivas encaminadas a hacer que los individuos y las colectividades estén en condiciones de ejercer un mayor control sobre los determinantes de su salud.

Con todo, en un contexto de aseguramiento que fundamentalmente piensa en acciones orientadas al individuo, se ha de asumir la llamada información, educación y comunicación como una estrategia central de la PS, pero acompañada de otras estrategias como el empoderamiento, el mercadeo social y la acción intersectorial. Esto es inevitable si lo que se busca es mantener la fidelidad a las propuestas de la PS.

Por lo pronto, es necesario convencer a las Empresas Promotoras de Salud-EPS que, aún en el contexto del SGSSS y con los limites que plantea el Plan Obligatorio de Salud-POS, deben pensar más allá de la enfermedad, más allá de la escueta acción asistencial sobre el individuo y más allá de la simple acción sectorial, con el fin de cumplir su función social de ser garantes del cumplimiento de las acciones de PS. No es tarea fácil, es cierto, pero es esencial para forjar una verdadera conciencia promocional y para cumplir 
con los objetivos de la seguridad social y dentro de ella, el principio de la protección integral •

Agradecimientos. Al equipo de trabajo de la Universidad Nacional de Colombia conformado por Jaime Cardona, Ernaldo Díaz, Gloria Garay, Alexander Gómez, Catalina Latorre y Rosa Suárez que, en el marco del proyecto del Ministerio de Salud "Ajuste al sistema de fortalecimiento de la gestión de las aseguradoras y las entidades territoriales respecto a las acciones de promoción y prevención del POS y el POS-S y las normas de gestión de los recursos disponibles para desarrollar acciones de promoción y prevención”, ha venido reflexionando sobre estos temas y dándole contenido a las propuestas de cambio.

\section{REFERENCIAS}

1. Unión Internacional de Promoción de la Salud y Educación para la Salud. La evidencia de la eficacia de la promoción de la salud. Libro de Evidencia (Parte Dos). Informe de la Unión Internacional de Promoción de la Salud y Educación para la Salud, para la Comisión Europea. Ministerio de Sanidad y Consumo, España; 2000.

2. De Salazar L. Promoción de la salud y evidencias de su efectividad: un reto metodológico y político. En: La promoción de la salud en América Latina: modelos, estructuras y visión crítica. Arroyo H (edit.). Universidad de Puerto Rico, San José: 39-750; 2004.

3. Thorogood M, Coombes Y. Evaluating Health Promotion: Practice and Methods. Great Britain, Oxford University Press; 2003.

4. Arroyo H. La promoción de la salud en América Latina: modelos, estructuras y visión crítica. San Juan: Universidad de Puerto Rico; 2004.

5. Pedersen A, O'Neill M, Rootman I. Health Promotion in Canada. Provincial, National \& International Perspectives. WB Saunders Canada. Canada;1994.

6. Restrepo H, Malaga H. Promoción de la Salud: Cómo construir vida saludable. Edit. Médica Panamericana, Bogotá ;2001.

7. Eslava JC. Promoción y prevención en el sistema de salud en Colombia. Revista de Salud Publica. 2002; 4 (1): 1-12.

8. Kickbusch I. Promoción de la salud: una perspectiva mundial. En: Promoción de la Salud: una antología. Washington, D.C.: OPS. Publicación Científica No. 557; 1996. pp 15-24.

9. Nutbeam D. Glosario de Promoción de la Salud. En: Promoción de la salud: una antología. Washington, D.C.: OPS. Publicación Científica No. 557; 1996. pp. 383-403. 1996.

10. Colomer C, Alvarez-Dardet C. Promoción de la salud y cambio social. Masson S.A., Barcelona.;2001.

11. Puska P, Nissinen A, Tuomilehto J, Salonen J, Koskela K, McAlister A et al. Estrategia basada en la comunidad para prevenir las cardiopatías isquémicas: conclusiones de los primeros 10 años del proyecto de Carelia del 
Norte. En: Promoción de la Salud: una antología. Washington, D.C.: OPS. Publicación Científica No. 557; 1996. pp 99-139.

12. Organización Mundial de la Salud. Fomento de la salud a través de la Escuela. Informe de un Comité de Expertos de la OMS. En: Educación sanitaria y Fomento de la salud integrales en las escuelas. Serie Informes Técnicos No. 870. Ginebra;1997.

13. Ministerio de Salud de Colombia. Municipios Saludables. Declaración de Santafé de Bogotá para la promoción de la salud. Bogotá: Min-Salud/ OPS; 1992.

14. Ministerio de Salud de Colombia. Promoción de la Salud. Bogotá: Min-Salud/ OPS; 1992.

15. Ministerio de Salud de Colombia. Conferencia Internacional de Promoción de la Salud. Memorias. Bogotá: Min-Salud-OPS-OMS; 1992. 